\title{
BMJ Open Prevalence of strabismus among preschool children in eastern China and comparison at a 5-year interval: a population-based cross-sectional study
}

Yue Wang, ${ }^{1,2}$ Andi Zhao, ${ }^{1,2}$ Xiaohan Zhang, ${ }^{3}$ Dan Huang, ${ }^{4}$ Hui Zhu, ${ }^{1,2}$ Qigang Sun, ${ }^{5}$ JiaJia Yu, ${ }^{6} \mathrm{Ji}$ Chen, ${ }^{5}$ Xiaoyan Zhao, ${ }^{7}$ Rui $\mathrm{Li}^{1,}{ }^{1,2}$ Shu Han, ${ }^{8}$ Weiyi Dong, ${ }^{9}$ Fanfei Ma, ${ }^{9}$ XueJuan Chen, ${ }^{1,2}$ Hu Liu (iD) ${ }^{1,2}$

To cite: Wang Y, Zhao A, Zhang $X$, et al. Prevalence of strabismus among preschool children in eastern China and comparison at a 5-year interval: a population-based crosssectional study. BMJ Open 2021;11:e055112. doi:10.1136/ bmjopen-2021-055112

- Prepublication history for this paper is available online. To view these files, please visit the journal online (http://dx.doi org/10.1136/bmjopen-2021055112).

YW and $A Z$ contributed equally.

YW and $A Z$ are joint first authors.

Received 03 July 2021

Accepted 21 September 2021

A) Check for updates

(C) Author(s) (or their employer(s)) 2021. Re-use permitted under CC BY-NC. No commercial re-use. See rights and permissions. Published by BMJ.

For numbered affiliations see end of article.

Correspondence to

Dr Hu Liu; liuhu@njmu.edu.cn

\section{ABSTRACT}

Objective To update data on strabismus and evaluate the changes in prevalence and patterns among preschoolers in eastern China over a period of 5 years.

Design Nanjing Eye Study, a longitudinal populationbased study.

Setting Recruitment and testing in kindergartens in Yuhuatai District, Nanjing.

Participants 2300 eligible children.

Main outcome measures Comprehensive ocular examinations were conducted in 1986 children aged 48- <60 months in Nanjing Eye Study (NES, 2016-2017), including visual acuity, ocular alignment, refractive error and ocular structures evaluation. The prevalence rate and pattern of strabismus were calculated and compared with those from the Nanjing Pediatric Vision Project (NPVP, 2011-2012) in children of the same age, of the same area and using the same diagnostic criteria.

Results The overall prevalence rate of strabismus in NES was $5.56 \%$ (95\% Cl 4.54\% to $6.57 \%)$, which was not significantly different from that in NPVP $(4.99 \%, 95 \% \mathrm{Cl}$ $4.13 \%$ to $5.84 \%, p=0.40$ ). The prevalence of subtypes of strabismus underwent significant changes, with significant increase in intermittent exotropia (IXT) in NES $(2.78 \%$ vs $4.69 \%, p=0.001$ ) and significant decrease in constant exotropia ( $1.17 \%$ vs $0.15 \%, p<0.001)$. Significant change in pattern was observed in IXT, where the proportion of the convergence insufficiency type (2.90\% vs $27.17 \%)$ increased and exceeded the divergence excess type (20.29\% vs $11.96 \%)$ to be the second common type $(p<0.001)$.

Conclusion The prevalence of strabismus appeared stable in children aged $48-<60$ months in eastern China at a 5-year interval. The prevalence of IXT increased significantly, and the convergence insufficiency type became more prevalent in patients with IXT. Timely detection and intervention of IXT are important among preschoolers.

\section{INTRODUCTION}

Strabismus is a common ocular disorder in childhood which can lead not only to cosmetic impairment but also to loss of binocularity and depth perception. ${ }^{1-5}$ The negative
Strengths and limitations of this study

- Studies that evaluate changes in the prevalence and characteristics of strabismus over time in preschool children are rare, and this study provides an update on data on strabismus in eastern China and reported the changes.

- This is a large population-based study comprising 1986 preschoolers aged $48-<60$ months.

- The study has a high participation rate $(>80 \%)$ and used the same standard procedures and diagnostic criteria in children of the same age and of the same area, allowing a direct comparison between two studies.

- Prevalence estimate might be biased due to poor cooperation from young children, and history of strabismus treatment as recalled by parents could be subject to recall bias.

impact of this early-onset visual developmental disorder can persist into adulthood if left untreated. ${ }^{6}$ Identifying strabismus at an earlier age may prevent the development of strabismic amblyopia, as well as improve the chance of restoring binocularity, highlighting the importance of early detection and timely interventions. ${ }^{7}$ A study on the epidemiology of strabismus would provide useful data to guide screening and control of strabismus at an early age.

So far, several population-based studies, including the Baltimore Pediatric Eye Disease Study, ${ }^{8}$ the Multiethnic Pediatric Eye Disease Study (MEPEDS) ${ }^{9}{ }^{10}$ the Strabismus, Amblyopia and Refractive Error in Singapore Study, ${ }^{11}$ and the Vision in Preschoolers Study, ${ }^{12}$ have reported the prevalence rate of strabismus to vary from $1 \%$ to $4.6 \%$ in preschoolers and revealed disparities in the prevalence and pattern in different regions of the world and ethnic populations. ${ }^{7-11}$ However, these 
cross-sectional studies only captured a snapshot of the ocular disorder at a single point in time. Due to changes in the natural environment, ${ }^{13}$ social economy, ${ }^{14}{ }^{15}$ lifestyle and vision care system, ${ }^{16}$ studies that evaluate changes in the prevalence and characteristics of ocular diseases over time are needed to help obtain valuable feedback for ongoing vision care efforts, bringing the next stage targeted approaches and improvement directions. This will help us accomplish the goal of eliminating the main causes of all preventable and treatable blindness set forth in 'Vision 2020'. ${ }^{17}$

In the Nanjing Pediatric Vision Project (NPVP) we carried out in 2011-2012, the prevalence rate of strabismus was $4.99 \%{ }^{18}$ In 2016, the Nanjing Eye Study (NES) was conducted in children of the same age and of the same area using the same study procedures and diagnostic criteria. In this study, we assessed the prevalence of strabismus and its subtypes in NES and compared it with NPVP. The aim of this study is to provide an update on the prevalence rate of strabismus and evaluate its changes among preschoolers in eastern China over a 5 -year period.

\section{METHODS}

\section{Study design and population}

NES is a population-based cohort eye study designed to longitudinally evaluate the initiation and progression of childhood ocular diseases by establishing a systematic database in eastern China. Of all children in Yuhuatai District, those born between September 2011 and August 2012 and at the point of entering kindergarten were invited to participate in this study to undergo a comprehensive eye examination annually. Data presented in this study were obtained from 2016 to 2017, when these children were 48-<60 months old. Among 2300 eligible preschoolers, 1986 (response rate $83.5 \%$ ) children participated in this study. ${ }^{19}{ }^{20}$ Written informed consent was obtained from the parents or legal guardians of all participants. Oral assent was obtained from all children right before the examination.

\section{Ocular examination}

In both NES and NPVP, a comprehensive eye examination was performed on children by a team composed of trained ophthalmologists and optometrists using similar protocols as MEPEDS, ${ }^{21}$ including anthropometric parameters, ocular biometric parameters, distance visual acuity, anterior segment, refractive error, stereoacuity test and ocular alignment and motility. The Hirschberg light reflex test was performed to assess ocular alignment at a distance of $33 \mathrm{~cm}$, followed by the cover-uncover test and the alternate cover test, with fixation targets at a distance of $33 \mathrm{~cm}$ and $6 \mathrm{~m}$, with or without correction (if worn). Monocular and binocular ocular movements were examined at nine diagnostic positions of gaze. An alternate prism cover test was used to detect the degree of eye misalignment in children with suspected strabismus. Once strabismus was suspected, a prism cover test was used to detect the degree of eye misalignment. If the child was suspected of having intermittent exotropia (IXT), one eye was covered with thick gauze to break the tenacious proximal fusion between the two eyes for at least $30 \mathrm{~min}$, and then an alternate prism cover test was performed to assess the degree of exotropia.

\section{Inclusion and exclusion criteria}

Only children aged $48-<60$ months who were able to complete the ocular alignment test were included in this analysis and considered as the general study population.

\section{Definitions}

Strabismus was defined as any tropia present at a distance or near, with or without spectacles, and then classified according to its primary direction (esotropia, exotropia, vertical). Strabismus is considered constant if constant both at near and distance fixation; otherwise it is considered intermittent. IXT was defined as an intermittent exodeviation of at least 10 prism dioptres (PD) at a distance. According to the Burian's classification, IXT is classified into four subtypes based on the difference between the distant $(6 \mathrm{~m})$ and near $(33 \mathrm{~cm})$ angles of deviation: 'basic type' (difference $<10 \mathrm{PD}$ ), 'convergence weakness type' (near angle minus distant angle $\geq 10 \mathrm{PD}$ ), 'pseudo divergence excess type' (distant angle minus near angle $\geq 10 \mathrm{PD}$ but $<10 \mathrm{PD}$ after $30 \mathrm{~min}$ monocular patching), and 'true divergence excess type' (distant angle minus near angle $\geq 10 \mathrm{PD}$ even after patching). ${ }^{22} \mathrm{In}$ this study, all IXT suspects had one of their eyes patched before the alternative prism cover test. Therefore, the 'pseudo divergence excess type' was counted in the 'basic type' category in this analysis. Microstrabismus was defined as a deviation of less than $10 \mathrm{PD}$, in addition to binocular vision. Children with a history of strabismus or treatment were regarded as strabismic.

\section{Statistical analyses}

Statistical analyses were performed using IBM SPSS V.13.0 (www.ibm.com). All statistical tests were twosided, and $\mathrm{p}<0.05$ was considered statistically significant. t-test was used to compare the means. The prevalence rates of strabismus and its subtypes were calculated for NES and NPVP separately and were compared between the two studies using $\chi^{2}$ test or Fisher's exact test. The 95\% CI for prevalence rates was calculated using normal approximation.

\section{Patient and public involvement}

Patients and the public were not involved in any aspects of the study, including the development of study question, study design, conduct of the study and dissemination of results.

\section{RESULTS}

Prevalence of strabismus in NES and comparison with NPVP Ultimately, 1961 (of 1986, 98.7\%) children with complete data were included in the sample for statistical analysis. 
Table 1 Prevalence and characteristics of strabismus in children of the Nanjing Eye Study $(n=1961)$

\begin{tabular}{|c|c|c|c|}
\hline Characteristics & Without strabismus (n) & With strabismus (n) & Prevalence rate, $\%(95 \% \mathrm{Cl})$ \\
\hline \multicolumn{4}{|l|}{ Gender } \\
\hline \multicolumn{4}{|l|}{ Any strabismus } \\
\hline Female & 841 & 45 & 5.08 (3.63 to 6.52$)$ \\
\hline Overall & 1852 & 109 & 5.56 (4.54 to 6.57$)$ \\
\hline Male & 1067 & 8 & $0.74(0.23$ to 1.26$)$ \\
\hline Female & 883 & 3 & 0.34 (0 to 0.72$)$ \\
\hline Overall & 1950 & 11 & $0.56(0.23$ to 0.89$)$ \\
\hline \multicolumn{4}{|l|}{ Concomitant exotropia } \\
\hline Male & 1021 & 54 & 5.02 (3.72 to 6.33$)$ \\
\hline \multicolumn{4}{|l|}{ Types } \\
\hline Concomitant strabismus & 1855 & 106 & 5.41 (4.40 to 6.41$)$ \\
\hline Esotropia & 1950 & 11 & $0.56(0.23$ to 0.89$)$ \\
\hline Constant & 1950 & 11 & 0.56 (0.23 to 0.89$)$ \\
\hline Intermittent & 1961 & 0 & 0 \\
\hline Exotropia & 1866 & 95 & 4.84 (3.89 to 5.79$)$ \\
\hline Constant & 1958 & 3 & 0.15 (0 to 0.33$)$ \\
\hline Intermittent & 1869 & 92 & 4.69 (3.76 to 5.63$)$ \\
\hline Basic & 1905 & 56 & 2.86 (2.12 to 3.59$)$ \\
\hline
\end{tabular}

The detailed characteristics of the children had been described in previous published articles. ${ }^{19} 20$

A total of 109 children were diagnosed with strabismus in NES, with an overall prevalence rate of $5.56 \%$ (95\% CI $4.54 \%$ to $6.57 \%)$. The prevalence of concomitant esotropia and concomitant exotropia was $0.56 \%$ (95\% CI $0.23 \%$ to $0.89 \%$ ) and $4.84 \%$ (95\% CI $3.89 \%$ to $5.79 \%$ ), respectively (table 1 ). There was no statistical gender difference in the overall prevalence of strabismus $(5.95 \%$ vs $5.08 \%, \mathrm{p}=0.40)$, concomitant esotropia $(0.74 \%$ vs $0.34 \%, \mathrm{p}=0.36)$ or concomitant exotropia $(5.02 \%$ vs $4.63 \%, \mathrm{p}=0.69)$.

Among 109 preschoolers with strabismus in NES, two presented normal ocular alignment but with history of strabismus surgery (1 esotropia and 1 exotropia preoperatively). In terms of the pattern of strabismus, 11 had concomitant esotropia, 95 had concomitant exotropia, 1 had pure vertical strabismus (superior oblique muscle palsy) and 2 had special forms of strabismus (1 Duane syndrome and 1 Brown syndrome). Four exotropia cases were combined with vertical deviation $(3.7 \%, 4$ of 109$)$.
The most frequent type of strabismus was IXT $(84.4 \%$, 92 of 109), followed by constant esotropia (10.1\%, 11 of 109), constant exotropia (2.8\%, 3 of 109) and special forms of strabismus $(1.8 \%, 2$ of 109$)$, while vertical deviation $(0.9 \%, 1$ of 109$)$ accounted for the least (figure 1).

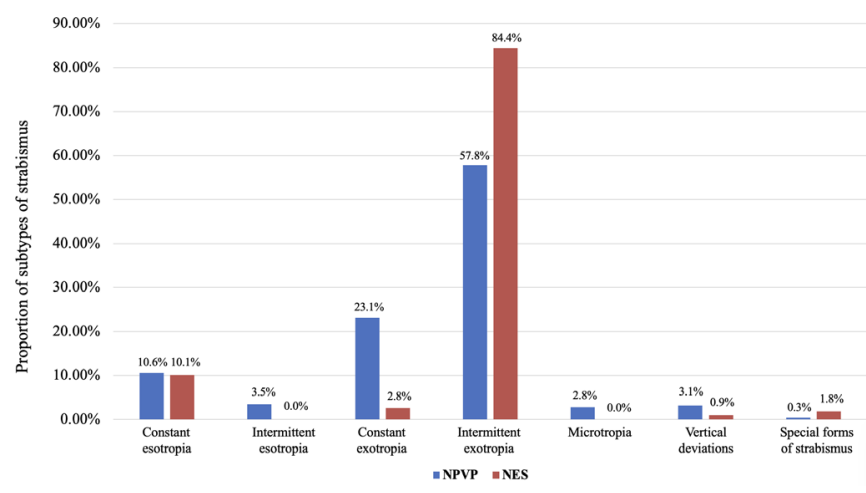

Figure 1 Proportion of subtypes of strabismus in the Nanjing Eye Study (NES) and in the Nanjing Pediatric Vision Project (NPVP). 
Table 2 Comparison of children with strabismus aged $48-<60$ months in the population of NPVP $(n=2486)$ and NES $(n=1961)$

\begin{tabular}{|c|c|c|c|}
\hline Characteristics & NPVP & NES & $P$ value \\
\hline Gender, n (\%) & & & 0.98 \\
\hline Male & $73(58.9)$ & $64(58.7)$ & \\
\hline Female & $51(41.1)$ & $45(41.3)$ & \\
\hline Prevalence of strabismus, \% & 4.99 & 5.56 & 0.40 \\
\hline Prevalence of concomitant esotropia, \% & 0.68 & 0.56 & 0.61 \\
\hline Prevalence of concomitant exotropia, \% & 3.94 & 4.84 & 0.14 \\
\hline Prevalence of constant exotropia, \% & 1.17 & 0.15 & $<0.001$ \\
\hline Prevalence of IXT, \% & 2.78 & 4.69 & 0.001 \\
\hline $\mathrm{XT}: \mathrm{ET}$ ratio & $5.8: 1$ & $8.6: 1$ & 0.33 \\
\hline Subtypes of concomitant horizontal strabismus, $\mathrm{n}(\%)$ & & & $<0.001^{*}$ \\
\hline Constant esotropia & $14(12.2)$ & $11(10.4)$ & \\
\hline Intermittent esotropia & $3(2.6)$ & $0(0)$ & \\
\hline Constant exotropia & $29(25.2)$ & $3(2.8)$ & \\
\hline IXT & $69(60.0)$ & $92(86.8)$ & \\
\hline Subtypes of IXT, n (\%) & & & $<0.001$ \\
\hline Divergence excess & $14(20.3)$ & $11(12.0)$ & \\
\hline Convergence weakness & $2(2.9)$ & $25(27.2)$ & \\
\hline Basic & $53(76.8)$ & $56(60.8)$ & \\
\hline Mean SER in children with strabismus, dioptre & $1.720 \pm 1.779$ & $0.971 \pm 1.863$ & 0.003 \\
\hline History of strabismus treatment, $\mathrm{n}(\%)$ & & & 0.78 \\
\hline Yes & $21(16.9)$ & $20(18.4)$ & \\
\hline No & $103(83.1)$ & $89(81.6)$ & \\
\hline History of esotropia treatment, $\mathrm{n}(\%)$ & & & $0.041^{*}$ \\
\hline Yes & $8(47.1)$ & $10(90.9)$ & \\
\hline No & $9(52.9)$ & $1(9.1)$ & \\
\hline History of exotropia treatment, $\mathrm{n}(\%)$ & & & 0.71 \\
\hline Yes & $12(12.2)$ & $10(10.5)$ & \\
\hline No & $86(87.8)$ & 85 (89.5) & \\
\hline
\end{tabular}

Bold print represents statistical significance.

${ }^{\star}$ Fisher's exact test.

ET, esotropia; IXT, intermittent exotropia; NES, Nanjing Eye Study; NPVP, Nanjing Pediatric Vision Project; SER, spherical equivalent refraction; XT, exotropia.

\section{Comparison between NES and NPVP}

Table 2 shows the comparison of the characteristics of strabismus between NPVP and NES children. The prevalence of strabismus $(\mathrm{p}=0.40)$ and the exotropia to esotropia ratio $(p=0.33)$ were similar between the two studies. Statistically significant difference was observed between the two studies in terms of the pattern of horizontal strabismus $(p<0.001)$, driven by the lower prevalence of constant exotropia in NES than in NPVP $(0.15 \%$ vs $1.17 \%, \mathrm{p}<0.001)$ and higher prevalence of IXT in NES than in NPVP $(4.69 \%$ vs $2.78 \%, \mathrm{p}=0.001)$. The proportion of the IXT subtype was different between the two studies $(\mathrm{p}<0.001)$, with convergence weakness accounting for a larger proportion in NES than in NPVP (27.2\% vs $2.9 \%, \mathrm{p}<0.001)$. Of 109 NES children with strabismus, 20 $(18.4 \%)$ were reported to have had previous treatment, which was similar to NPVP $(16.9 \%, \mathrm{p}=0.78)$. Treatment rate was higher in NES than in NPVP for esotropia $(90.9 \%$ vs $47.1 \%, \mathrm{p}=0.04)$, while it remained stable for exotropia ( $10.5 \%$ vs $12.2 \%, \mathrm{p}=0.71)$. The mean $\pm \mathrm{SD}$ spherical equivalent refraction (SER) in children with strabismus was $0.97 \pm 1.86$ dioptres (D) in NES and $1.72 \pm 1.78 \mathrm{D}$ in NPVP and their difference was statistically significant $(p=0.003)$.

\section{DISCUSSION}

In this population-based NES study, we reported an overall prevalence rate of strabismus of $5.56 \%$ in children aged $48-<60$ months in eastern China. The overall concomitant esotropia and exotropia prevalence rates are $0.56 \%$ and $4.84 \%$, respectively, with IXT as the most common type of strabismus. Compared with our NPVP study 
conducted 5 years ago using the same study protocol in children of the same area as the NES study, no significant difference was found in the prevalence of strabismus. However, the prevalence of IXT was significantly higher in NES, whereas the prevalence of constant exotropia was only one-tenth of that reported in NPVP.

Both NPVP and NES had high participation rates $(>80 \%)$ and used the same diagnostic criteria as MEPEDS, allowing a direct comparison between them to evaluate changes in strabismus in Chinese preschool children. Our data showed that the prevalence of strabismus appeared stable over the past 5 years. An Israeli investigation found a decline in the prevalence of young adulthood strabismus from 1974 to 1994, which might be due to better diagnosis and treatment in the paediatric population. ${ }^{23}$ Taken into consideration that our analyses did not exclude those who had been successfully treated, we believe that our results tended to reflect the natural change in the experience of strabismus in this area. Our previous study found that the rate of reduced Uncorrected visual acuity (UCVA) increased significantly in a 5-year interval, which was likely due to the influence of some environmental risk factors, such as spending more time on mobile devices. ${ }^{19}$ Conversely, the steady trend in the rate of strabismus in the present study indicated that genetic factors might play a more important role in the occurrence of strabismus in early life.

The prevalence of strabismus in NPVP or NES in children aged 48-<60 months old was similar to those reported in other studies. However, the exotropia to esotropia ratio was highest in NES (8.6:1) and NPVP (5.8:1) among the investigations (table 3), which is in line with the high exotropia to esotropia ratio reported by several studies based on East Asian populations. ${ }^{112425}$ This ratio has increased over the past 5 years, from 5.8 in NPVP to 8.6 in NES, but their difference was not significant. Nevertheless, the proportion of exotropia underwent significant change. An increase in the proportion of patients with IXT was observed, while constant exotropia became less common in NES. A previous Hong Kong study reported a similar increasing trend in IXT accompanied by an apparent decline in esotropia. On the contrary, the prevalence of esotropia appeared stable in our study. A Hong Kong study attributed this change to the increasing prevalence of myopia. ${ }^{26}$ We observed a declining trend in SER in that children with strabismus in NES were less hyperopic than those in NPVP $(0.97 \mathrm{D}$ vs $1.72 \mathrm{D}, \mathrm{p}=0.003)$. Given that myopia is not the predominant refractive error in preschoolers with strabismus, ${ }^{27}$ this explanation may not be appropriate in our study. Taken together the opposite change in the prevalence of IXT and constant exotropia, we speculated that regular vision screening and timely intervention might reduce the risk of deterioration of IXT and thus lead to the number of patients with constant exotropia decreased. In addition, improved pregnant and prenatal care might play a role as well. ${ }^{28}{ }^{29}$ It is noteworthy that the prevalence of esotropia was lower than those reported in other studies (table 3) and showed a slight decrease over the past 5 years. Besides the widespread ethnic and regional disparities, early significant hyperopia correction likely played a role in preventing children with hyperopia from developing esotropia in this population.

IXT was found to be the most common subtype in both NPVP and NES. Notably, the proportion of convergence insufficiency increased and exceeded divergence excess to be the second major type of IXT in NES. So far, few population-based studies had studied the subtypes of IXT. Since the increased prevalence of IXT had been found in this area, investigations on its causes and characteristics are of great importance to broaden our understanding of IXT. Chia et al, ${ }^{30}$ in their hospital-based

Table 3 Prevalence of amblyopia and strabismus in children aged $48-<60$ months from population-based or large cohort studies

\begin{tabular}{|c|c|c|c|c|c|c|c|c|c|}
\hline \multirow[b]{2}{*}{ Study } & \multirow[b]{2}{*}{ Year of study } & \multirow[b]{2}{*}{ Country } & \multirow[b]{2}{*}{ Ethnicity } & \multirow{2}{*}{$\begin{array}{l}\text { Sample } \\
\text { size }\end{array}$} & \multicolumn{5}{|c|}{ Prevalence rate $(\%)$ of strabismus } \\
\hline & & & & & Total & $\mathbf{X T}$ & ET & IXT & XT:ET \\
\hline \multirow[t]{2}{*}{ MEPEDS } & 2003-2011 & USA & Hispanic/Latino & 539 & 3.90 & 2.04 & 1.5 & NA & $1.4: 1$ \\
\hline & & & $\begin{array}{l}\text { Non-Hispanic } \\
\text { white }\end{array}$ & 271 & 4.06 & 1.48 & 2.58 & NA & $1: 1.8$ \\
\hline BPEDS & & & African American & 261 & 2.30 & 0.77 & 1.53 & NA & $1: 2$ \\
\hline STARS & 2006-2008 & Singapore & Chinese & 602 & 1.17 & 1.17 & 0 & NA & NA \\
\hline NPVP & 2011-2012 & China & Chinese & 2486 & 4.99 & 3.94 & 0.68 & 2.78 & $5.8: 1$ \\
\hline NES & 2016-2017 & China & Chinese & 1961 & 5.56 & 4.84 & 0.56 & 4.69 & $8.6: 1$ \\
\hline
\end{tabular}

BPEDS, Baltimore Pediatric Eye Disease Study; ET, esotropia; IXT, intermittent exotropia; MEPEDS, Multiethnic Pediatric Eye Disease Study; NA, not applicable; NES, Nanjing Eye Study; NPVP, Nanjing Pediatric Vision Project; STARS, Strabismus, Amblyopia and Refractive Error in Singapore; XT, exotropia. 
study, reported that divergence excess IXT was the major subtype. However, the lack of patching in Chia $e$ t $a l \mathrm{~s}^{30}$ methodology might overestimate the amount of cases of divergence excess. All children in NPVP and NES suspected with IXT had one of their eyes patched before examinations, increasing the accuracy of IXT classification by weakening the influence of tenacious proximal fusion. ${ }^{31}$ Consistent with our study, a Korean hospitalbased investigation also found convergence insufficiency as the second common subtype of IXT. ${ }^{32}$ The reason for changes in the pattern of IXT is unknown and future investigation on the causes and characteristics of IXT is warranted.

NPVP and NES had similar treatment rates for strabismus, with almost one in five children with strabismus receiving treatment before our examination. So far, few studies have directly reported treatment rates, making comparison with other studies difficult. Although no significant difference between NES and NPVP was found in the overall treatment rate, a significant increase in esotropia treatment rate was found. Vision screening in preschoolers in the Yuhuatai District was performed earlier than other communities in China. Early detection and timely referral had achieved good outcomes, which would contribute to the rise in the treatment rate of esotropia. Given early diagnosis and treatment are associated with better outcome in strabismus, more efforts ought to be made on comprehensive screening, timely referral and treatment.

The strengths of this study include the large and population-based sample of preschoolers aged $48-<60$ months old in two studies from the same area that followed standardised protocols for comprehensive eye examinations and used the same diagnostic criteria, allowing us to reliably assess the change in prevalence in strabismus at a 5 -year interval.

This study had a few limitations. First, poor cooperation from these very young children may lead to underestimation of the prevalence rate of strabismus. Second, history of treatment and pattern of strabismus with previous treatment were determined by medical records or were recalled by the parents, with the latter subject to recall bias.

\section{CONCLUSION}

In summary, the NES found an overall prevalence rate of strabismus of $5.56 \%$ in children aged $48-<60$ months in eastern China. The prevalence of strabismus appeared stable, but the pattern of strabismus underwent significant changes over the past 5 years, comparable with our previous NPVP study. The prevalence of IXT increased significantly, and the high exotropia to esotropia ratio was the most noteworthy feature in this area. More efforts on vision screening should be made in order to detect and provide intervention for IXT in the future.

\section{Author affiliations}

${ }^{1}$ Department of Ophthalmology, The First Affiliated Hospital with Nanjing Medical University, Nanjing, Jiangsu, China

${ }^{2}$ Nanjing Medical University, Nanjing, Jiangsu, China

${ }^{3}$ Ophthalmology, Wuxi Children's Hospital, Wuxi, China

${ }^{4}$ Department of Child Healthcare, The First Affiliated Hospital with Nanjing Medical University, Nanjing, China

${ }^{5}$ Ophthalmology, Maternal and Child Healthcare Hospital of Yuhuatai District Nanjing China, Nanjing, Jiangsu, China

${ }^{6}$ Ophthalmology, Wuxi No 2 People's Hospital, Wuxi, Jiangsu, China

${ }^{7}$ Ophthalmology, The Affiliated Changzhou No 2 People's Hospital of Nanjing Medical University, Nanjing, Jiangsu, China

${ }^{8}$ Ophthalmology, Soochow University Affiliated Children's Hospital, Suzhou, Jiangsu, China

${ }^{9}$ Fourth School of Clinical Medicine of Nanjing Medical University, Nanjing, China

Acknowledgements We thank the children, their parents or legal guardians, and all the members of the Maternal and Child Healthcare Hospital of Yuhuatai District, Nanjing, China, for their helpful advice and support.

Contributors $\mathrm{HL}$ and XJC designed the study. YW, XZhang and AZ wrote the main manuscript text. YW, XZhang and RL prepared the tables. YW, XZhang, DH, SH, HZ and XZhao performed data interpretation and analysis. YW, XZhang, QS, JC, JJY, WD and FM performed the ocular examinations. $\mathrm{HL}$ is responsible for the overall content as guarantor.

Funding This work was supported by the National Natural Science Foundation of China (grant no 81673198 and no 81803258), the Natural Science Foundation of Jiangsu Province (grant no BK20161595), Jiangsu Province's Key Provincial Talents Programme (grant no QNRC2016563), and the Postgraduate Research and Practice Innovation Programme of Jiangsu Province (grant no SJCX20_0477).

Competing interests None declared.

Patient and public involvement Patients and/or the public were not involved in the design, or conduct, or reporting, or dissemination plans of this research.

Patient consent for publication Not required.

Ethics approval This study was approved by the ethics committee of the First Affiliated Hospital with Nanjing Medical University (2017-SR-205) in accordance with the Declaration of Helsinki principles.

Provenance and peer review Not commissioned; externally peer reviewed.

Data availability statement All data relevant to the study are included in the article or uploaded as supplementary information.

Open access This is an open access article distributed in accordance with the Creative Commons Attribution Non Commercial (CC BY-NC 4.0) license, which permits others to distribute, remix, adapt, build upon this work non-commercially, and license their derivative works on different terms, provided the original work is properly cited, appropriate credit is given, any changes made indicated, and the use is non-commercial. See: http://creativecommons.org/licenses/by-nc/4.0/.

ORCID iD

Hu Liu http://orcid.org/0000-0002-3952-4165

\section{REFERENCES}

1 Sharma P, Gaur N, Phuljhele S, et al. What's new for us in strabismus? Indian J Ophthalmol 2017;65:184.

2 Carlton J, Kaltenthaler E. Health-related quality of life measures (HRQoL) in patients with amblyopia and strabismus: a systematic review. Br J Ophthalmol 2011;95:325-30.

3 Blair E, Smithers-Sheedy H, Australian Cerebral Palsy Register Group. Strabismus, a preventable barrier to social participation: a short report. Dev Med Child Neurol 2016;58 Suppl 2:57-9.

4 Hatt SR, Leske DA, Castañeda YS, et al. Association of strabismus with functional vision and Eye-Related quality of life in children. JAMA Ophthalmol 2020;138:528-35.

5 Zipori AB, Colpa L, Wong AMF, et al. Postural stability and visual impairment: assessing balance in children with strabismus and amblyopia. PLoS One 2018;13:e0205857.

6 Thorn F, . Development of refraction and strabismus. Curr Opin Ophthalmol 2000;11:301-5.

7 Smith EL, Hung L-F, Arumugam B, et al. Observations on the relationship between anisometropia, amblyopia and strabismus. Vision Res 2017;134:26-42. 
8 Friedman DS, Repka MX, Katz J, et al. Prevalence of amblyopia and strabismus in white and African American children aged 6 through 71 months the Baltimore pediatric eye disease study. Ophthalmology 2009;116:2128-34.

9 Multi-ethnic Pediatric Eye Disease Study Group. Prevalence of amblyopia and strabismus in African American and Hispanic children ages 6 to 72 months the multi-ethnic pediatric eye disease study. Ophthalmology 2008;115:1229-37.

10 McKean-Cowdin R, Cotter SA, Tarczy-Hornoch K, et al. Prevalence of amblyopia or strabismus in asian and non-Hispanic white preschool children: multi-ethnic pediatric eye disease study. Ophthalmology 2013;120:2117.

11 Chia A, Dirani M, Chan Y-H, et al. Prevalence of amblyopia and strabismus in young singaporean Chinese children. Invest Ophthalmol Vis Sci 2010;51:3411-7.

12 Ying G-shuang, Maguire MG, Cyert LA, et al. Prevalence of vision disorders by racial and ethnic group among children participating in head start. Ophthalmology 2014;121:630-6.

13 Fleischer NL, Merialdi M, van Donkelaar A, et al. Outdoor air pollution, preterm birth, and low birth weight: analysis of the world Health organization global survey on maternal and perinatal health. Environ Health Perspect 2014;122:425-30.

14 Mojon-Azzi SM, Kunz A, Mojon DS. The perception of strabismus by children and adults. Graefes Arch Clin Exp Ophthalmol 2011;249:753-7.

15 Graham H, White PCL. Social determinants and lifestyles: integrating environmental and public health perspectives. Public Health 2016;141:270-8

16 Wang J, Li M, Zhu D, et al. Smartphone overuse and visual impairment in children and young adults: systematic review and meta-analysis. J Med Internet Res 2020;22:e21923.

17 Bourne RRA. Vision 2020: where are we? Curr Opin Ophthalmol 2020;31:81-4.

18 Chen X, Fu Z, Yu J, et al. Prevalence of amblyopia and strabismus in Eastern China: results from screening of preschool children aged 36-72 months. Br J Ophthalmol 2016;100:515-9.

19 Zhang $X$, Wang Y, Huang D, et al. Prevalence of reduced visual acuity among preschool children in eastern China and comparison at a 5year interval. Clin Exp Ophthalmol 2018;46:994-1001.
20 Han S, Zhang X, Zhao X, et al. Stereoacuity and related factors in healthy preschool children: the Nanjing eye study. Ophthalmic Epidemiol 2019;26:336-44.

21 Varma R, Deneen J, Cotter S, et al. The multi-ethnic pediatric eye disease study: design and methods. Ophthalmic Epidemiol 2006;13:253-62.

22 Burian HM. Exodeviations: their classification, diagnosis and treatment. Am J Ophthalmol 1966;62:1161-6.

23 Shapira Y, Machluf Y, Mimouni M, et al. Amblyopia and strabismus: trends in prevalence and risk factors among young adults in Israel. $\mathrm{Br}$ J Ophthalmol 2018;102:659-66.

24 Goseki T, Ishikawa $\mathrm{H}$. The prevalence and types of strabismus, and average of stereopsis in Japanese adults. Jpn J Ophthalmol 2017;61:280-5.

$25 \mathrm{Han}$ KE, Baek S-H, Kim S-H, et al. Prevalence and risk factors of strabismus in children and adolescents in South Korea: Korea National health and nutrition examination survey, 2008-2011. PLoS One 2018;13:e0191857-11.

26 Yu CBO, Fan DSP, Wong VWY, et al. Changing patterns of strabismus: a decade of experience in Hong Kong. Br J Ophthalmol 2002;86:854-6.

27 Guo X, Fu M, Ding X, et al. Significant axial elongation with minimal change in refraction in 3- to 6-year-old Chinese preschoolers: the Shenzhen kindergarten eye study. Ophthalmology 2017;124:1826-38.

28 Yang Y, Wang C, Gan Y, et al. Maternal smoking during pregnancy and the risk of strabismus in offspring: a meta-analysis. Acta Ophthalmol 2019;97:353-63.

29 Torp-Pedersen T, Boyd HA, Poulsen G, et al. In-Utero exposure to smoking, alcohol, coffee, and tea and risk of strabismus. Am J Epidemiol 2010;171:868-75.

30 Chia A, Roy L, Seenyen L. Comitant horizontal strabismus: an Asian perspective. Br J Ophthalmol 2007;91:1337-40.

31 Pan C-W, Zhu H, Yu J-J, et al. Epidemiology of intermittent exotropia in preschool children in China. Optom Vis Sci 2016;93:57-62.

32 Jung J-W, Lee S-Y. A comparison of the clinical characteristics of intermittent exotropia in children and adults. Korean $J$ Ophthalmol 2010;24:96-100. 